\title{
Platelet Storage Results in a Redistribution of Glycoprotein Ib Molecules Evidence for a Large Intraplatelet Pool of Glycoprotein lb
}

\author{
Alan D. Michelson, Burt Adelman, * Marc R. Barnard, Eddie Carroll, and Robert I. Handin \\ Department of Pediatrics, University of Massachusetts Medical School, Worcester, Massachusetts 01655; ${ }^{*}$ Departments of Medicine, \\ Medical College of Virginia and Hunter Holmes McGuire Veterans Administration Hospital, Richmond, Virginia 23298; \\ and ${ }^{\ddagger}$ Hematology Division, Brigham and Women’s Hospital, and Department of Medicine, Harvard Medical School, \\ Boston, Massachusetts 02115
}

\begin{abstract}
Platelet membrane glycoprotein (GP) Ib contains receptors for von Willebrand factor and thrombin. Its proteolytic fragment, glycocalicin, circulates in normal plasma. In this study, storage of platelet concentrates for $5 \mathrm{~d}$ resulted in a $221 \%$ increase in plasma glycocalicin (1.3 times the total amount of glycocalicin present on the surface of all platelets), an $8 \%$ overall increase in platelet surface GPIb, and the appearance of a surface GPIb-negative subpopulation of platelets. Total platelet GPIb content of fresh washed platelets, determined by gel electrophoresis and immunoassay of Triton X-100 lysates, averaged 159,740 molecules per platelet. There were 36,360 surface GPIb molecules per platelet, determined by immunoassay of the supernatant of fresh washed platelets whose surface GPIb had been completely plasmin-cleaved. In summary, these studies provide evidence for $(a)$ a redistribution of GPIb molecules with platelet storage, and (b) a large intraplatelet pool of GPIb (approximately threefold larger than the platelet surface pool of GPIb).
\end{abstract}

\section{Introduction}

The platelet surface membrane contains an estimated 26,000 34,000 glycoprotein $(\mathrm{GP})^{1}$ Ib molecules per platelet $(1,2)$. Platelet surface GPIb contains receptors for the plasma proteins, von Willebrand factor $(1,3)$ and thrombin $(4,5)$. Binding of von Willebrand factor to its receptor on platelet surface GPIb is important in the initiation of the process of platelet adhesion to damaged blood vessel walls (1). Binding of thrombin to its receptor(s) on platelet surface GPIb is important in the initiation of the process of platelet activation $(4,5)$. Glycocalicin, a proteolytic fragment of the $\alpha$-chain of GPIb, circulates in normal plasma at a concentration of $1-3 \mu \mathrm{g} / \mathrm{ml}(6)$.

This work was presented in part at the 29th Annual Meeting of the American Society of Hematology and published in abstract form (1987. Blood. 70:355a).

Address reprint requests to Dr. Michelson, Department of Pediatrics, University of Massachusetts Medical School, 55 Lake Avenue North, Worcester, MA 01655.

Received for publication 28 August 1986 and in revised form 17 December 1987.

1. Abbreviations used in this paper: GP, glycoprotein; RSF, relative specific fluorescence.

J. Clin. Invest.

(C) The American Society for Clinical Investigation, Inc. 0021-9738/88/06/1734/07 \$2.00

Volume 81, June 1988, 1734-1740
The glycocalicin fragment contains the GPIb receptor site for von Willebrand factor $(7)$ and thrombin $(4,5)$.

In addition to the platelet surface and plasma pools of GPIb, there may be a third intraplatelet pool of GPIb. Both Asch et al. (8) and Wencel-Drake et al. (9), using frozen thinsection immunoelectron microscopy, have recently described an intraplatelet pool of GPIb, located on the intraluminal face of vacuolar structures (9). However, this putative intraplatelet pool of GPIb has not been quantitated or functionally characterized.

If the present study, we demonstrate that platelet storage results in a redistribution of the platelet surface and plasma pools of GPIb. This redistribution suggested the presence of a large intraplatelet pool of GPIb, which we have now quantitated.

\section{Methods}

Monoclonal antibodies. We utilized three murine monoclonal antibodies (6D1, WM23, and 3G6) directed against the glycocalicin portion of the $\alpha$-chain of platelet membrane GPIb. 6D1 and WM23 were generously provided by Drs. Barry S. Coller (SUNY, Stony Brook, NY) and Michael C. Berndt (University of Sydney, Australia), respectively. 3G6 was prepared in our laboratory by standard hybridoma technology (10) after injection of mice with glycocalicin, purified as previously described (11). 6D1 is directed at the von Willebrand factor binding site on the glycocalicin portion of the $\alpha$-chain of GPIb (3). WM23 and 3G6 are also directed against the glycocalicin portion of the $\alpha$-chain of GPIb, but not against the von Willebrand factor binding site (Berndt et al. [12] and B. Adelman, unpublished observations). WM23 has been characterized as being directed against the macroglycopeptide portion of glycocalicin, rather than the terminal protein portion that contains the von Willebrand factor and 6D1 binding sites (M. C. Berndt, personal communication). Unlike 6D1, both $3 \mathrm{G} 6$ and WM23 recognize their respective antigens in Western blots. After complete platelet solubilization with sodium dodecyl sulfate (SDS) polyacrylamide gel electrophoresis (PAGE) and Western blotting, both 3G6 and WM23 (M. C. Berndt, personal communication) recognize only a single band (GPIb) in both reduced and nonreduced gels. As a control antibody in the flow cytometry assay, we utilized OX6 (provided by Dr. Bruce Woda, University of Massachusetts Medical School), a mouse monoclonal antibody of the same IgG subtype as 6D1 and WM23, but directed at rat lymphocyte $I_{\mathrm{a}}$ antigen.

Platelet concentrate preparation and storage. Blood from five different normal donors was collected into citrate-phosphate-dextrose solution and individual platelet concentrates, resuspended in $\sim 50 \mathrm{ml}$ of their own plasma, were prepared in PL-732 bags (Fenwal Laboratories, Morton Grove, IL) by standard methods (13). After being left stationary at $22^{\circ} \mathrm{C}$ for $1 \mathrm{~h}$, the platelet concentrates were stored in a linear platelet reciprocator model LPR-1 (Melco Engineering Corp., Glendale, CA) with gentle horizontal agitation at $70 \mathrm{rpm}$ for $5 \mathrm{~d}$ at $22^{\circ} \mathrm{C}$. Immediately after preparation (day 0 ) and then daily for $5 \mathrm{~d}$, samples from the platelet concentrates were aseptically withdrawn through a sample port into a syringe containing EDTA and aprotinin 
(Sigma Chemical Co., St Louis, MO) (final concentrations: EDTA 10 $\mathrm{mM}$, aprotinin $1,000 \mathrm{KIU} / \mathrm{ml})$. For all samples from all donors, each assay (flow cytometric analysis of platelet surface GPIb, enzyme-linked immunosorbent assay (ELISA) for plasma glycocalicin, and platelet count) was performed at the same time using the same reagents. There was no platelet clumping in the concentrates, as determined both by direct inspection and phase microscopy performed daily for the $5 \mathrm{~d}$ of storage. All donors were normal adult volunteers who had given written informed consent for blood collection.

Platelet counts. Platelet counting was performed in a Sysmex automated hematology analyzer model CC-800 (Toa Medical Electronics, Carson, CA).

Flow cytometric analysis of platelet surface GPIb. Daily samples removed from the platelet concentrates were fixed in $1 \%$ formaldehyde for $30 \mathrm{~min}$ at $22^{\circ} \mathrm{C}$. As previously described (14-16), platelets were washed, incubated with either a saturating concentration of monoclonal antibody 6D1 or the irrelevant monoclonal antibody OX6, washed again, incubated with a saturating concentration of fluorescein isothiocyanate-labeled goat anti-mouse IgG antibody (Cooper Biomedical, Malvern, PA), washed again, and analyzed in a FACS 440 (Becton Dickinson FACS Systems, Mountain View, CA). For each sample the fluorescence signal from 10,000 individual cells was measured. Three-dimensional contour maps were ungated. All histograms were gated by forward light scatter to include $90 \%$ of cells (excluding $\sim 5 \%$ of both the smallest and largest cells), with the approximate median being determined by $2-\mu \mathrm{m}$ diam plastic beads (Polysciences, Inc., Warrington, PA). Based on their characteristic forward and orthogonal light scatter profiles (and as confirmed by appropriate monoclonal antibodies), no red cells, lymphocytes, monocytes, or granulocytes were among the cells assessed in any of the gated histograms or ungated contour maps. Results were expressed in one of three ways: $(a)$ the number of platelets with a completely negative surface GPIb phenotype, as determined by the percentage of platelets that, after incubation with monoclonal antibody 6D1 followed by goat anti-mouse antibody, did not have fluorescence $>95 \%$ of platelets incubated with monoclonal antibody OX6 followed by goat antimouse antibody; $(b)$ relative specific fluorescence (RSF) (arbitrary linear units), calculated from the mean channel number of all platelets, as previously described (15); (c) RSF of surface GPIb-positive platelets, calculated as previously described (15) but using the mean channel number of only those platelets that, after incubation with $6 \mathrm{D} 1$ followed by goat antimouse antibody, had fluorescence $>95 \%$ of platelets incubated with OX6 followed by goat anti-mouse antibody.

ELISA for plasma glycocalicin. Daily samples removed from the platelet concentrates were centrifuged at $2,000 \mathrm{~g}$ for $10 \mathrm{~min}$ at $22^{\circ} \mathrm{C}$ and the upper two-thirds of the plasma was removed. In control assays, the plasma was centrifuged at $22^{\circ} \mathrm{C}$ for an additional $2 \mathrm{~h}$ at $100,000 \mathrm{~g}$. All plasma samples were stored at $-70^{\circ} \mathrm{C}$ until assayed. After thawing, the samples were centrifuged again at $8,000 \mathrm{~g}$ for $4 \mathrm{~min}$ at $22^{\circ} \mathrm{C}$. The final plasma supernatants were assessed for glycocalicin content by ELISA, as previously described $(7,11)$. Briefly, a monolayer of platelets was fixed to plastic microwells and the ability of diluted plasma samples to inhibit the binding of monoclonal antibody $6 \mathrm{D} 1$ to platelet surface GPIb was compared to a standard curve of the inhibition of monoclonal antibody 6D1 binding by known nanomolar concentrations of purified glycocalicin. Parallel assays were performed in which 6D1 was replaced by either monoclonal antibody WM23 or monoclonal antibody 3G6. All assays were performed in triplicate.

Total platelet GPIb content determined by ELISA of $1 \%$ Triton $X$-100-solubilized platelets. Blood from normal donors was drawn directly into a syringe containing $1 / 6 \mathrm{vol}$ of acid-citrate-dextrose (trisodium citrate $85 \mathrm{mM}$, citric acid $71 \mathrm{mM}$, dextrose $111 \mathrm{mM}$, $\mathrm{pH} 4.5$ ), resulting in a final $\mathrm{pH}$ of 6.5 , as previously described (17). The blood was centrifuged at $150 \mathrm{~g}$ for $15 \mathrm{~min}$ at $22^{\circ} \mathrm{C}$ and the platelet-rich plasma separated. The platelets were washed twice in Tyrode's buffer (138 mM NaCl, $29 \mathrm{mM} \mathrm{KCl}, 12 \mathrm{mM} \mathrm{NaHCO}{ }_{3}, 0.4 \mathrm{mM} \mathrm{NaHPO}_{4}$, $0.1 \%$ glucose, $0.35 \%$ bovine serum albumin), $\mathrm{pH} 6.5$, containing 50 $\mathrm{ng} / \mathrm{ml} \mathrm{PGE}_{1}$, and resuspended in Tyrode's buffer, $\mathrm{pH} 7.4$, containing

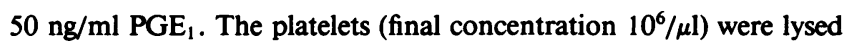
by addition of an equal volume of buffer containing $2 \%$ Triton X-100 (Sigma Chemical Co.), $100 \mathrm{mM}$ Tris- $\mathrm{HCl}$, pH 7.4, with $2 \mathrm{mg} / \mathrm{ml}$ leupeptin (Sigma Chemical Co.), as described by Fox et al. (18). The samples were then centrifuged at $8,000 \mathrm{~g}$ for $4 \mathrm{~min}$, conditions that do not sediment the majority of actin filament-associated GPIb (Fox [18] and see below). The resulting supernatants were assessed by ELISA (as described above) for their ability to inhibit either monoclonal antibody $6 \mathrm{D} 1$ or monoclonal antibody WM23 binding to platelet surface GPIb. Each platelet lysate was assayed at two dilutions, each in triplicate. Platelet lysates from the patient with Bernard-Soulier syndrome were adjusted to a protein concentration equivalent to that of normal platelet lysates, as determined by the bicinchoninic acid protein assay (Pierce Chemical Co., Rockford, IL) performed as previously described (19).

Total platelet GPIb content determined by SDS-PAGE. As described above, platelets were separated from the blood of normal donors, washed twice in Tyrode's buffer, $\mathrm{pH} 6.5$, containing $50 \mathrm{ng} / \mathrm{ml}$ $\mathrm{PGE}_{1}$, and resuspended in Tyrode's buffer, $\mathrm{pH}$ 7.4. SDS-PAGE (5\% polyacrylamide) of platelet pellets was performed according to the method of Laemmli (20). For each of six normal donors, the following samples were run on a single gel: four dilutions of their whole platelets (nonreduced); a standard curve of known amounts of glycocalicin (nonreduced) (purified from multiple donors as previously described [7]); a standard curve of known molecular weight standards (Bethesda Research Laboratories, Rockville, MD). The gels were stained with periodic acid-Schiff's reagent. Densitometry using a Quick Scan $\mathbf{R}+\mathbf{D}$ (Helena Laboratories, Beaumont, TX) was performed in triplicate. The individual peaks corresponding to the GPIb bands (the most heavily glycosylated; mol wt 163,400 ) of the whole platelet samples, and the glycocalicin bands ( $\mathrm{mol}$ wt 127,600) of the standards, were cut out and weighed. The value for each peak was averaged from the three scans. The total platelet protein content was determined to be 2.2 $\times 10^{-12} \pm 0.1 \times 10^{-12} \mathrm{~g} /$ platelet (mean \pm SEM, $n=10$ ) by the bicinchoninic acid protein assay (19). The amount of GPIb in the whole platelet samples was calculated by comparison to the standard curve and use of the following assumptions: (a) Molecular weight of GPIb was 163,400 and of glycocalicin 127,600 (by comparison to the known molecular weight standards). (b) The total carbohydrate content of GPIb is in the glycocalicin portion $(21,22)$. 3) The protein/carbohydrate ratio and the degree of glycosylation are the same for platelet surface GPIb and the putative intraplatelet pool of GPIb (suggested by the fact that monoclonal antibodies WM23 and 3G6 recognize only a single band (GPIb) on both Coomassie- and periodic acid-Schiffstained Western blots of solubilized platelets).

Plasmin treatment of washed platelets. Fresh platelets were washed as previously described (11), resuspended at a concentration of 1.0 $\times 10^{6} / \mu \mathrm{l}$ in Tris-EDTA-buffered saline $(10 \mathrm{mM}$ Tris, $0.15 \mathrm{M} \mathrm{NaCl}, 20$ mM EDTA, pH 7.4), and incubated for $1 \mathrm{~h}$ at $22^{\circ} \mathrm{C}$ with or without 1 casseinolytic unit/ml of plasmin (KABI through Helena Laboratories). The reaction was stopped by adding aprotinin in excess of the amount needed to totally inhibit plasmin activity (11). The platelet suspensions were then centrifuged $\left(2,000 \mathrm{~g}, 10 \mathrm{~min}, 22^{\circ} \mathrm{C}\right)$ and the glycocalicin content of the supernatants determined by ELISA, as described above. The platelet pellets were resuspended in Tris-EDTA-buffered saline and fixed overnight at $4^{\circ} \mathrm{C}$ in $1 \%$ formaldehyde. The fixed platelets were washed three times in Tris-buffered saline $(2,000 \mathrm{~g}, 10 \mathrm{~min}$, $22^{\circ} \mathrm{C}$ ) and resuspended in a concentration of $25,000 / \mu 1$. Plasmintreated and non-plasmin-treated platelets were then analyzed by flow cytometry for content of surface GPIb, as described above.

Bernard-Soulier syndrome patient. M.R. is a 26-yr-old woman with the Bernard-Soulier syndrome, as previously described (15).

\section{Results}

Fig. 1 shows the effect of length of storage of platelet concentrates on plasma glycocalicin concentration, as measured by 


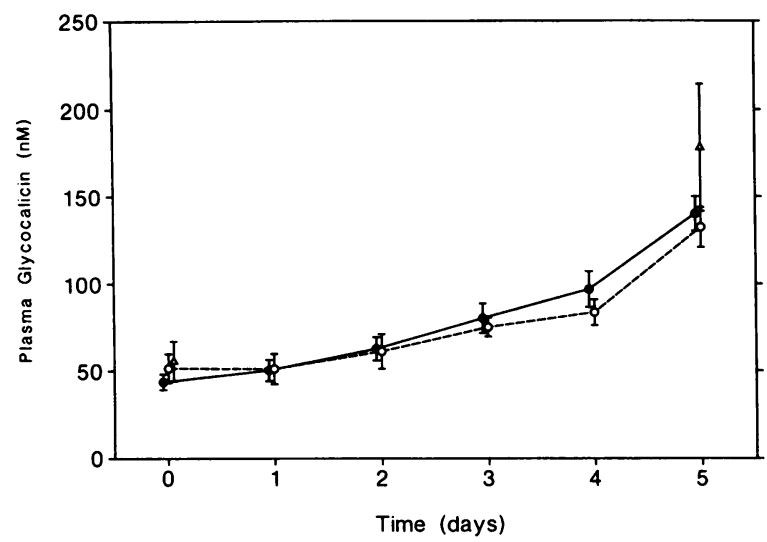

Figure 1. Effect of length of storage of platelet concentrates on plasma glycocalicin concentration, as determined by ELISA and monoclonal antibodies 6D1 (-, ๑), WM23 (- - , o), and 3G6 $(\Delta)$. Mean \pm SEM for five different donors analyzed simultaneously.

ELISA. Between days 0 and 5 there was a $221 \%$ increase in plasma glycocalicin, as determined by monoclonal antibody 6D1. Comparable results were obtained in parallel assays in which monoclonal antibodies WM23 or $3 \mathrm{G} 6$ were used rather than 6D1 (Fig. 1). This marked rise in plasma glycocalicin was not the result of any of the following, for the reasons stated: $(a)$ Platelet lysis: There was no significant change in platelet count per microliter between day $0 \quad(1,212,000 \pm 205,000$ [mean \pm SEM, $n=5$ ] ) and day $5(1,131,000 \pm 184,000)$. (b) Residual platelets or shedding of platelet membrane microparticles (23): An additional centrifugation of day 5 plasma at $100,000 \mathrm{~g}$ (i.e., at forces in excess of those required to sediment platelets and microparticles [23]) resulted in no significant change $(2.2 \pm 7.7 \%$ [mean \pm SEM] decrease) in the glycocalicin concentration of the supernatant. (c) Proteases derived from contaminating bacteria in the platelet concentrates (24): Microbiological cultures of all day 5 platelet concentrates were sterile. (d) In vitro manipulation of samples after their withdrawal from the platelet concentrate bags: We deliberately used conditions similar to those for which Coller et al. (6) demonstrated no in vitro cleavage of glycocalicin, and on day 0 we detected a plasma glycocalicin level (as determined by monoclonal antibody 6D1) of $43.6 \pm 4.6 \mathrm{nM}(2.18 \pm 0.23 \mu \mathrm{g}$ of protein $/ \mathrm{ml})($ mean \pm SEM, $n=5)$ (Fig. 1), which is similar to the plasma glycocalicin level of $1-3 \mu \mathrm{g} / \mathrm{ml}$ that Coller et al. (6) detected in normal plasma using the same antibody. (e) Further proteolysis of glycocalicin in plasma: We have previously demonstrated that proteolytic digestion of glycocalicin does not interfere with the epitope recognized by monoclonal antibody 6D1 and does not alter the quantity of glycocalicin detected by the ELISA (11).

Three lines of evidence suggested that the ELISA was correctly quantitating plasma glycocalicin. First, the glycocalicin concentration of normal donor plasma and purified glycocalicin was the same whether the samples were quantitated separately or in a mixture. Secondly, as stated above, we obtained plasma glycocalicin levels on day 0 which were comparable to those obtained in normal donors by Coller et al. (6). Thirdly, in separate experiments, treatment of washed platelets $(1.0$ $\times 10^{6} / \mu \mathrm{l}$ ) with plasmin 1 casseinolytic unit/ml for $1 \mathrm{~h}$ at $22^{\circ} \mathrm{C}$ resulted in complete cleavage of platelet surface GPIb, as quantitated by antibody 6D1 and flow cytometry (as pre- viously described [14]), and release of $36,360 \pm 1,390$ (mean \pm SEM, $n=4$ ) molecules of glycocalicin per platelet, as quantitated by antibody 6D1 in the ELISA (Table I). This number is consistent with the number of platelet surface GPIb molecules determined by other investigators $(1,2)$.

Assuming then that there were initially 36,360 molecules of GPIb on the surface of each platelet, the observed increase in plasma glycocalicin concentration between days 0 and 5 corresponded to 1.3 times the total amount of glycocalicin present on the surface of all the platelets. Fig. 2 shows the effect of length of storage on the relative number of surface GPIb molecules per platelet, as determined by monoclonal antibody 6D1 and flow cytometry. Rather than a loss, after $24 \mathrm{~h}$ of storage we observed a $25 \%$ increase in the number of surface GPIb molecules per platelet (Fig. 2: solid circles, solid line). Over the ensuing $4 \mathrm{~d}$ of storage, the number of surface GPIb molecules per platelet gradually decreased, but by day 5 of storage there was still an $8 \%$ increase in the number of surface GPIb molecules per platelet compared with day 0 (Fig. 2: solid circles, solid line).

The flow cytometric method enabled us to determine that the storage-related increase in the total number of platelet surface GPIb molecules per platelet (Fig. 2) was not the result of a uniform increase in the number of GPIb molecules on all platelets. As shown in Fig. 3, storage of platelets resulted in the appearance of two phenotypically distinct subpopulations of platelets: one surface GPIb-positive, the other surface GPIbnegative. With increasing length of storage, platelets with the surface GPIb-negative phenotype comprised an increasing proportion of the total platelet number, comprising $\sim 12 \%$ of all platelets by day 5 (Fig. $4 \mathrm{~A}$ ). After $24 \mathrm{~h}$, platelets with the surface GPIb-positive phenotype had a $28 \%$ increase in the number of surface GPIb molecules, as determined by flow cytometry (Fig. $4 B$ ). Over the ensuing $4 \mathrm{~d}$ of storage, platelets with the surface GPIb-positive phenotype gradually expressed less surface GPIb molecules, but by day 5 of storage there was still a $16 \%$ increase in the number of surface GPIb molecules compared with this subpopulation of platelets on day 0 (Fig. 4 $B)$. Using the formula: $\operatorname{RSF}_{\text {pred }}=(100-z) / 100 \times \mathrm{RSF}_{\text {pos }}$, where RSF $_{\text {pred }}$ is the predicted RSF for all platelets, $z$ is the percentage of platelets with a completely negative surface GPIb phenotype, and RSF $_{\text {pos }}$ is the RSF of the GPIb-positive platelets, we determined that the increase in the number of surface GPIb molecules in the GPIb-positive subpopulation of

Table I. Number of GPIb Molecules per Platelet*

\begin{tabular}{lcc}
\hline & $\begin{array}{c}\text { Number of GPIb } \\
\text { molecules per platelet } \\
\text { (mean } \pm \text { SEM) }\end{array}$ & $\begin{array}{c}\text { Number } \\
\text { of donors }\end{array}$ \\
\hline Whole platelet & & \\
SDS-PAGE method & $151,800 \pm 3,500$ & 6 \\
ELISA (antibody 6D1) & $182,330 \pm 6,970$ & 9 \\
ELISA (antibody WM23) & $145,090 \pm 6,050$ & 9 \\
Platelet surface & & \\
ELISA (antibody 6D1) & $36,360 \pm 1,390$ & 4 \\
\hline
\end{tabular}

* Assays described in text.

₹ Determined from 1\% Triton X-100-solubilized platelets.

${ }^{8}$ Determined from supernatant of platelets treated with plasmin 1 casseinolytic unit/ml for $1 \mathrm{~h}$ at $22^{\circ} \mathrm{C}$. 


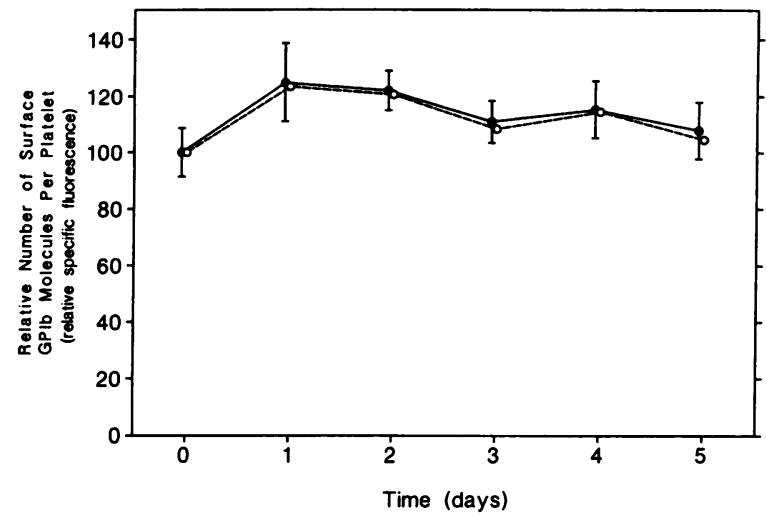

Figure 2. Effect of length of storage of platelet concentrates on the relative number of surface GPIb molecules per platelet, as determined by flow cytometry. At each time point for each of the five different donors, RSF was calculated from the mean channel number of 10,000 individually analyzed platelets incubated with monoclonal antibody 6D1 followed by fluoresceinated goat anti-mouse antibody, minus the mean channel number of 10,000 individually analyzed platelets incubated with an irrelevant monoclonal antibody followed by fluoresceinated goat anti-mouse antibody. Day 0 was arbitrarily assigned $100 \mathrm{U}$ of fluorescence. Mean \pm SEM for five different donors analyzed simultaneously. The observed data are shown as the solid circles and solid line. The values predicted from Fig. 4 (see Results) are shown as the open circles and dashed line.

platelets, together with the complete lack of surface GPIb molecules in the GPIb-negative subpopulation of platelets, fully accounted for the observed increase in the total number of surface GPIb molecules per platelet (Fig. 2: observed data are shown as the closed circles and solid line; predicted values are shown as the open circles and dashed line). Two additional pieces of information demonstrated by Fig. 3 are that: $(a)$ the forward light scatter of the platelets decreased with storage, suggesting that platelet size decreases with storage, and $(b)$ the forward light scatter of the surface GPIb-negative subpopulation of platelets was less than that of the surface GPIb-positive subpopulation of platelets, suggesting that the surface GPIbnegative platelets were smaller than the surface GPIb-positive platelets.

The unexpectedly large increase in plasma glycocalicin concentration during storage of platelet concentrates, in the face of the lack of an overall decrease in platelet surface GPIb, suggested the existence of an additional large intraplatelet pool of GPIb. In order to quantitate this putative intraplatelet pool of GPIb, the total platelet GPIb content was determined by SDS-PAGE. Solubilized whole platelets were compared to a standard curve of known amounts of purified glycocalicin (Fig. 5). The total number of GPIb molecules per platelet detected by SDS-PAGE was $151,800 \pm 3,500$ (mean \pm SEM, $n=6$ ) (Table I). The total number of GPIb molecules per platelet was therefore approximately fourfold greater than the number of GPIb molecules present on the platelet surface (Table I), suggesting the presence of an intraplatelet pool of GPIb which was approximately threefold larger than the platelet surface pool of GPIb.

In order to further substantiate our finding that the total platelet GPIb content was so much greater than the platelet surface pool, we quantitated the total platelet GPIb content by a different method: ELISA of $1 \%$ Triton X-100-solubilized platelets. The total number of GPIb molecules per platelet detected by ELISA using 6D1 (the monoclonal antibody directed against the von Willebrand factor binding site on GPIb) was $182,330 \pm 6,970(n=9)$. The total number of GPIb molecules per platelet detected by ELISA using WM23 (the monoclonal antibody directed against the macroglycopeptide portion of the $\alpha$-chain of GPIb) was $145,090 \pm 6,050(n=9)$ (Table I).

In the assay for total platelet GPIb content determined by ELISA of Triton X-100 solubilized platelets, the following three control experiments were performed: (a) replacement of platelets with buffer only, then treating with $1 \%$ Triton $X-100$, resulted in the detection of no GPIb $(n=3)$, thereby demonstrating that, at the dilutions used, Triton X-100 did not interfere with the ELISA; $(b)$ treatment of normal donor plasma with $1 \%$ Triton X-100 resulted in a plasma glycocalicin level,
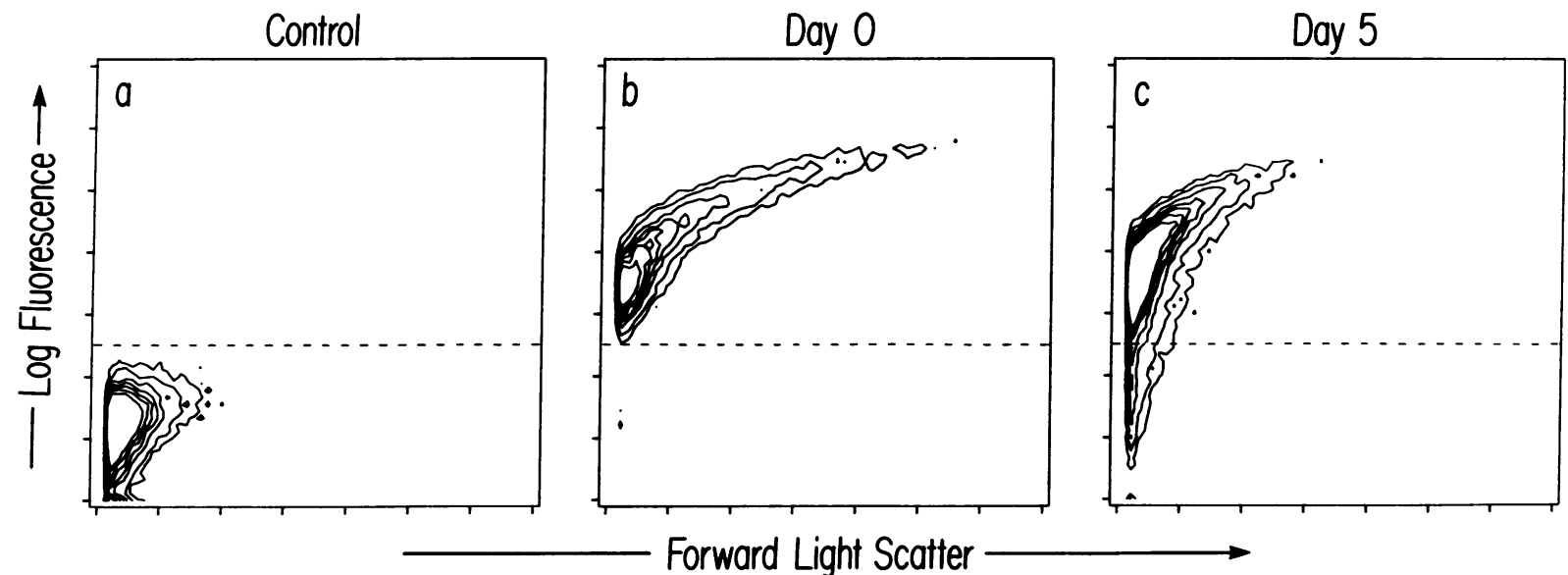

Figure 3. Effect of length of storage of platelet concentrates on the number of platelet surface GPIb molecules per platelet, as demonstrated by three-dimensional contour maps obtained by flow cytometry. The vertical axis is a logarithmic scale of fluorescence that corresponds to number of platelet surface GPIb molecules per platelet. The horizontal axis is a linear scale of forward light scatter that cor- responds to platelet size. The contour lines represent numbers of platelets, with the innermost contours corresponding to the highest numbers of platelets. Each map was obtained from 10,000 individually analyzed platelets from the same platelet concentrate. (a) Irrelevant monoclonal antibody. ( $b$ and $c$ ) GPIb-specific monoclonal antibody (6D1) with platelets from days 0 and 5 , respectively. 

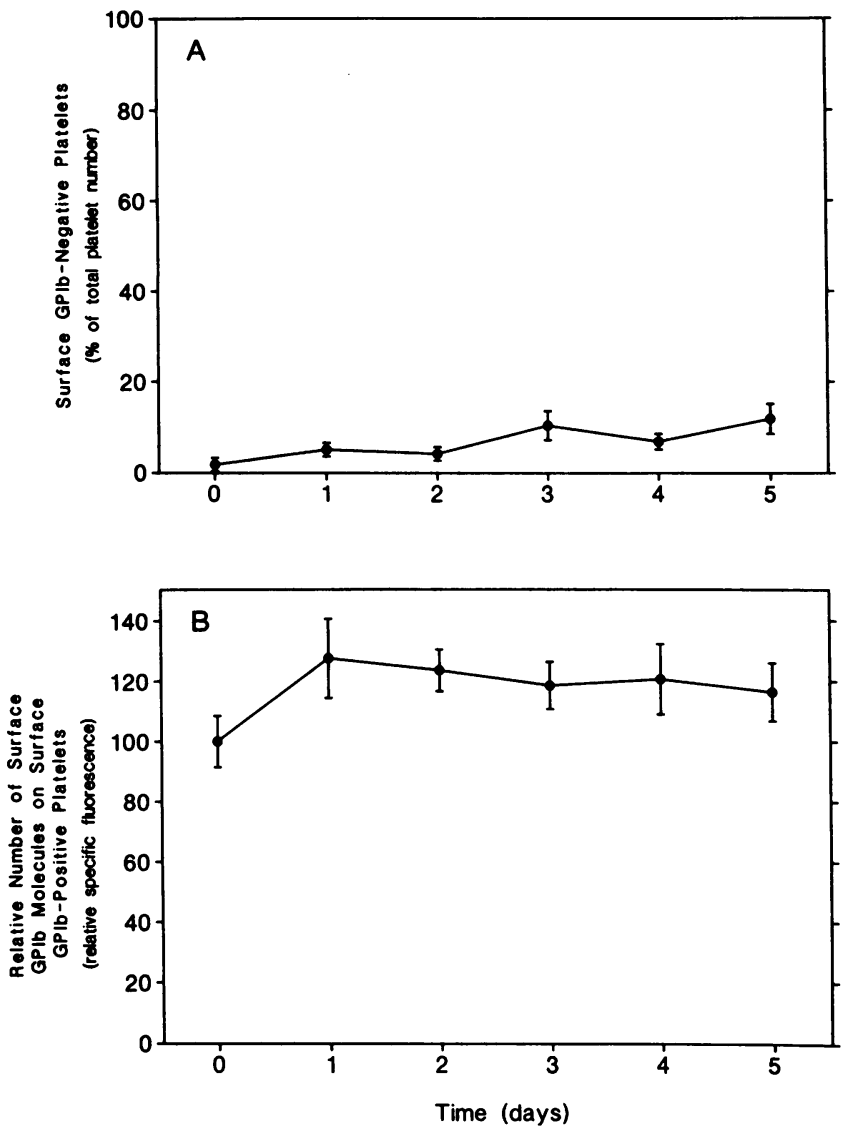

Figure 4. Effect of length of storage of platelet concentrates on surface GPIb-positive and surface GPIb-negative subpopulations of platelets, as determined by flow cytometry. Surface GPIb-negative platelets were defined as those platelets that, after incubation with 6D1 followed by goat anti-mouse antibody, did not have fluorescence $>95 \%$ of platelets incubated with an irrelevant monoclonal antibody followed by goat anti-mouse antibody. The remainder of the platelets were defined as surface GPIb-positive. $(A)$ Surface GPIbnegative platelets, expressed as a percentage of the total number of platelets. (B) Relative number of surface GPIb molecules on surface GPIb-positive platelets, as determined by RSF (calculated as in Fig. 2). Mean \pm SEM for five different donors analyzed simultaneously.

as determined by $6 \mathrm{D} 1$, which was $94.4 \pm 10.5 \%$ (mean \pm SEM, $n$ $=3$ ) of the plasma glycocalicin level of untreated normal plasma, thereby demonstrating that $1 \%$ Triton X-100 treatment of glycocalicin did not reveal additional epitopes recognizable by $6 \mathrm{Dl}$; $(c)$ within the range $1-10 \mathrm{nM}$, addition of various concentrations of purified glycocalicin to the Triton $\mathrm{X}-100$ platelet lysates had the predicted additive effect on the GPIb concentration. All platelet lysate samples were therefore assayed at a dilution within this range.

Fox $(18,25)$ found that $60-70 \%$ of platelet GPIb is bound to actin filaments and that, after $1 \%$ Triton $X-100$ platelet solubilization, high-speed centrifugation is required for their sedimentation. We therefore studied the effect of centrifugation conditions on quantitation of GPIb in whole-platelet lysates. With all other assay conditions kept constant, changing the centrifugation conditions of $1 \%$ Triton X-100 platelet lysates from our standard $(8,000 \mathrm{~g}$ for $4 \mathrm{~min})$ to those of Fox (18) $(100,000 \mathrm{~g}$ for $3 \mathrm{~h}$ ) resulted in a $52.8 \pm 4.4 \%$ (mean \pm SEM, $n$
=4) decrease in the total platelet GPIb content, determined by ELISA from the supernatants.

In order to provide additional evidence that no intraplatelet factor other than GPIb was interfering with the ELISA for total platelet GPIb content, we studied a patient (M.R.) with the Bernard-Soulier syndrome. Four serially diluted samples of her platelet lysates were assessed in the ELISA using both monoclonal antibody 6D1 and monoclonal antibody WM23. All assays resulted in undetectable levels of GPIb. Given that GPIb, GPV, and GPIX are the only deficiencies known to be present in the platelets of patients with the Bernard-Soulier syndrome (26), our data indicate that other intraplatelet factors do not interfere with the ELISA.

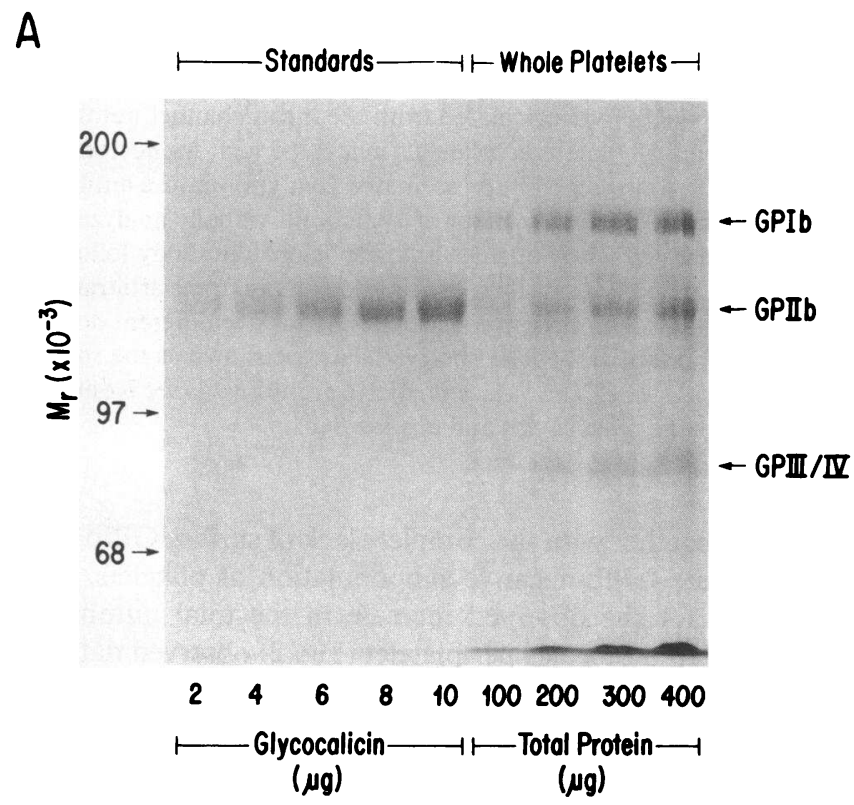

Figure 5. Total platelet GPIb content, as determined by SDS-PAGE. (A) Different amounts of solubilized whole platelets were compared to a standard curve of known amounts of purified glycocalicin. All samples were nonreduced. Gel was stained with periodic acid-Schiff's reagent. Densitometric peaks corresponding to the GPIb band (the most heavily glycosylated; $M_{\mathrm{r}} 163,400$ ) of the whole-platelet samples, and the glycocalicin band $\left(M_{\mathrm{r}} 127,600\right)$ of the standards, were cut out and weighed. $(B)$ Standard curve of known amounts of purified glycocalicin. The line of best fit was calculated by linear regression analysis. 


\section{Discussion}

This study demonstrates that platelet storage results in a redistribution of GPIb molecules, and provides evidence for a large intraplatelet pool of GPIb. Assuming a total platelet protein mass of between $1.7 \times 10^{-12}$ and $3.85 \times 10^{-12} \mathrm{~g}$ per platelet (27), our data suggest that between $0.6 \%$ and $1.5 \%$ of the total platelet protein mass is GPIb, making GPIb one of the most abundaht platelet proteins.

The presently described evidence for a large intraplatelet pool of GPIb is consistent with the findings of other investigators. Asch et al. (8) and Wencel-Drake et al. (9) used frozen thin-section immunoelectron microscopy to demonstrate an intraplatelet pool of GPIb which is distinct from the platelet surface pool of GPIb. Solum et al. (28) solubilized platelets by freeze-thawing and, using immunoelectrophoresis, found $67 \pm 22 \mu \mathrm{g}$ of glycocalicin $/ 10^{9}$ platelets. This corresponds to $287,143 \pm 94,286$ total GPIb molecules per platelet, a number comparable to those obtained in our experiments.

This study does not provide information about the location of the intraplatelet stores of GPIb. However, our previous work (16) suggests that intraplatelet GPIb is not contained in $\alpha$-granules. Whereas thrombin activation is associated with an increased platelet surface expression of platelet activation-dependent granule-external membrane (PADGEM) protein (a component of the $\alpha$-granule membrane of resting platelets) and release from platelets of $\beta$-thromboglobulin and platelet factor 4 (constituents of $\alpha$-granules), it is associated neither with an increased platelet surface expression of GPIb nor with release of GPIb from platelets (16).

In the ELISA measurement of total platelet GPIb content, our calculations assume that one molecule of GPIb and one molecule of glycocalicin each bind only one molecule of 6D1 antibody. With regard to the possibility that a repeating epitope for antibody $6 \mathrm{D} 1$ is revealed by solubilization and perhaps unfolding of the extensive carbohydrate portion of GPIb, we make the following points: 1 . The epitope for monoclonal antibody $6 \mathrm{D} 1$ is not in the carbohydrate portion of GPIb, because 6D1 binding to glycocalicin is not altered by $(a)$ the removal from glycocalicin of all its sialic acid and galactose residues and part of its $N$-acetyl glucosamine residues (7), or (b) the complete deglycosylation of glycocalicin (R. I. Handin, unpublished observations). 2 . We have previously demonstrated that proteolytic digestion of glycocalicin does not reveal additional epitopes recognized by $6 \mathrm{D} 1$ (11). 3 . In the present experiments we determined that $1 \%$ Triton X-100 treatment of glycocalicin did not reveal additional epitopes recognizable by 6D1 or WM23. 4. Comparable results for the total platelet GPIb content were obtained with antibodies 6D1 and WM23, despite each antibody being directed against a different part of the GPIb molecule.

Fox $(18,25)$ reported that $60-70 \%$ of platelet GPIb is bound to actin filaments present in the platelet skeleton. Fox (18) demonstrated that, after $1 \%$ Triton X-100 solubilization of platelets in the presence of $1 \mathrm{mg} / \mathrm{ml}$ leupeptin, the actin filament-associated GPIb is recovered mainly with those filaments which require high-speed centrifugation $(100,000 \mathrm{~g}$ for $3 \mathrm{~h}$ ) for their sedimentation, whereas low-speed centrifugation $(15,600 \mathrm{~g}$ for $4 \mathrm{~min})$ results in most of the GPIb being in the supernatant. Likewise, Solum and Olsen (29) demonstrated that solubilization of platelets with $1 \%$ Triton $\mathrm{X}-100$ in the presence of leupeptin resulted in essentially all the GPIb being in the supernatant after low-speed centrifugation $(8,000 \mathrm{~g}$ for 4 min) of the lysate. Consistent with the data of both Fox and Solum and their co-workers, we found that, after $1 \%$ Triton $\mathrm{X}-100$ solubilization of platelets in the presence of $1 \mathrm{mg} / \mathrm{ml}$ leupeptin, alteration of the centrifugation conditions of the platelet lysates from our standard $(8,000 \mathrm{~g}$ for $4 \mathrm{~min})$ to those of Fox (18) $(100,000 \mathrm{~g}$ for $3 \mathrm{~h})$ resulted in a $52.8 \pm 4.4 \%$ (mean \pm SEM, $n=4$ ) decrease in the GPIb content of the supernatant, as determined by ELISA. We conclude that our low-speed centrifugation conditions do not sediment the majority of actin filament-associated GPIb and that ELISA of this supernatant assays most of the total platelet GPIb content.

The present studies did not enable us to determine that the surface GPIb-negative phenotype was the result of a complete deficiency of platelet surface GPIb. A partial deficiency of the platelet surface GPIb molecule or a more subtle biochemical modification of the antibody binding site are alternative explanations for the lack of binding of monoclonal antibody $6 \mathrm{D} 1$ to the subpopulation of platelets with the surface GPIb-negative phenotype. Although thrombin can result in decreased binding of 6D1 to platelets (16), generation of thrombin would not account for the marked increase in plasma glycocalicin (16).

The present data are relevant to the clinical infusion of platelet concentrates into patients, because $(a)$ monoclonal antibody $6 \mathrm{D} 1$ is directed against the GPIb receptor for von Willebrand factor (3), $(b)$ this receptor has a central role in platelet adhesion to damaged blood vessel walls (1), and (c) our experiments were performed on platelet concentrates prepared exactly as for clinical transfusion purposes (13). Studies by other investigators of the GPIb content of stored platelet concentrates have yielded apparently conflicting results. In two separate radioligand-binding assays utilizing a monoclonal antibody (AN51) directed at GPIb and Maclura pomifera lectin, respectively, Taylor and Anstee (30) found no decrease in the surface GPIb content of stored platelet concentrates after 3 $\mathrm{d}$ of storage at $22^{\circ} \mathrm{C}$. Using SDS-PAGE of whole platelets and periodic acid Schiff's reagent staining, George (31) and Bolin et al. (32) found a $49 \%$ and a $29 \%$ decrease, respectively, in the total GPIb content of stored platelet concentrates after $3 \mathrm{~d}$ of storage at $22^{\circ} \mathrm{C}$. The data of all these investigators (30-32) are, however, consistent with the present finding of a redistribution of GPIb molecules.

\section{Acknowledgments}

The authors thank Drs. Barry Coller (SUNY, Stony Brook, NY) and Michael Berndt (University of Sydney, Australia) for generously providing antibodies 6D1 and WM23, respectively, Dr. Irma Szymanski for preparing the platelet concentrates, and Marcia McFadden and Julie Reitter for operating the flow cytometer.

Dr. Michelson was supported by FIRST Award HL-38138 and New Investigator Research Award HL-36809 from the National Institutes of Health, a grant from the American Heart Association, Massachusetts Affiliate, Inc., Basil O'Connor Starter Scholar Research Award No. 5-582 from the March of Dimes Birth Defects Foundation, and a Charles A. King Trust Research Fellowship from The Medical Foundation, Inc., Boston, MA. Dr. Adelman was supported by a Merit Review Grant from the Veterans Administration and a grant from The Council for Tobacco Research-U.S.A., Inc. Dr. Handin was supported by grants HL-34787 and HL-33014 from the National Institutes of Health. 


\section{References}

1. George, J. N., A. T. Nurden, and D. R. Phillips. 1984. Molecular defects in interactions of platelets with the vessel wall. N. Engl. J. Med. 311:1084-1098.

2. Coller, B. S. 1984. Report of the working party on hybridomaderived monoclonal antibodies to platelets. Thromb. Haemostasis. 51:169-173.

3. Coller, B. S., E. I. Peerschke, L. E. Scudder, and C. A. Sullivan. 1983. Studies with a murine monoclonal antibody that abolishes ristocetin-induced binding of von Willebrand factor to platelets: additional evidence in support of GPIb as a platelet receptor for von Willebrand factor. Blood. 61:99-110.

4. Takamatsu, J., M. K. Horne, and H. K. Gralnick. 1986. Identification of the thrombin receptor on human platelets by chemical crosslinking. J. Clin. Invest. 77:362-368.

5. Harmon, J. T., and G. A. Jamieson. 1986. The glycocalicin portion of platelet glycoprotein Ib expresses both high and moderate affinity receptor sites for thrombin. J. Biol. Chem. 261:13224-13229.

6. Coller, B. S., E. Kalomiris, M. Steinberg, and L. E. Scudder. 1984. Evidence that glycocalicin circulates in normal plasma. J. Clin. Invest. 73:794-799.

7. Michelson, A. D., J. Loscalzo, B. Melnick, B. S. Coller, and R. I. Handin. 1986. Partial characterization of a binding site for von Willebrand factor on glycocalicin. Blood. 67:19-26.

8. Asch, A. S., L. L. K. Leung, M. J. Polley, and R. L. Nachman. 1985. Platelet membrane topography: Colocalization of thrombospondin and fibrinogen with the glycoprotein IIb-IIIa complex. Blood. 66:926-934.

9. Wencel-Drake, J. D., E. F. Plow, T. J. Kunicki, V. L. Woods, D. M. Keller, and M. H. Ginsberg. 1986. Localization of internal pools of membrane glycoproteins involved in platelet adhesive responses Am. J. Pathol. 124:324-334.

10. Kohler, G., and C. Milstein. 1975. Continuous culture of fused cells secreting antibody of predefined specificity. Nature (Lond.). 256:495-497.

11. Adelman, B., A. D. Michelson, J. Loscalzo, J. Greenberg, and R. I. Handin. 1985. Plasmin effect on platelet glycoprotein Ib-von Willebrand factor interactions. Blood. 65:32-40.

12. Berndt, M. C., C. Gregory, A. Kabral, H, Zola, D. Fournier, and P. A. Castaldi. 1985. Purification and preliminary characterization of the glycoprotein Ib complex in the human platelet membrane. Eur. J. Biochem. 151:637-649.

13. Technical Manual of the American Association of Blood Banks. 1985. American Association of Blood Banks, Arlington, VA. 57-58.

14. Adelman, B., A. D. Michelson, R. I. Handin, and K. A. Ault. 1985. Evaluation of platelet glycoprotein Ib by fluorescence flow cytometry. Blood. 66:423-427.

15. Michelson, A. D. 1987. Flow cytometric analysis of platelet surface glycoproteins: Phenotypically distinct subpopulations of platelets in children with chronic myeloid leukemia. J. Lab. Clin. Med. 110:346-354.

16. Michelson, A. D., and M. R. Barnard. 1987. Thrombin-induced changes in platelet membrane glycoproteins Ib, IX, and IIb-IIIa complex. Blood. 70:1673-1678.
17. Kinlough-Rathbone, R. L., M. A. Packham, and J. F. Mustard. 1983. Platelet aggregation. In Measurements of Platelet Function. L. A. Harker and T. S. Zimmerman, editors. Churchill Livingstone, New York. 64-91.

18. Fox, J. E. B. 1985. Linkage of a membrane skeleton to integral membrane glycoproteins in human platelets: identification of one of the glycoproteins as glycoprotein Ib. J. Clin. Invest. 76:1673-1683.

19. Smith, P. K., R. I. Krohn, G. T. Hermanson, A. K. Mallia, F. H. Gartner, M. D. Provenzano, E. K. Fujimoto, N. M. Goeke, B. J. Olson, and D. C. Klenk. 1985. Measurement of protein using bicinchoninic acid. Anal. Biochem. 150:76-85.

20. Laemmli, U. K. 1970. Cleavage of structural proteins during the assembly of the head of bacteriophage T4. Nature (Lond.). 227:680-685.

21. Lopez, J. A., D. W. Chung, K. Fujikawa, F. S. Hagen, T. Papayannopoulou, and G. J. Roth. 1987. Cloning of the $\alpha$ chain of human platelet glycoprotein Ib: A transmembrane protein with homology to leucine-rich $\alpha_{2}$-glycoprotein. Proc. Natl. Acad. Sci. USA. 84:5615-5619.

22. Titani, K., K. Takio, M. Handa, and Z. M. Ruggeri. 1987. Amino acid sequence of the von Willebrand factor-binding domain of platelet membrane glycoprotein Ib. Proc. Natl. Acad. Sci. USA. 84:5610-5614.

23. George, J. N., L. L. Thoi, L. M. McManus, and T. A. Reimann. 1982. Isolation of human platelet membrane microparticles from plasma and serum. Blood. 60:834-840.

24. Cooper, H. A., W. P. Bennett, A. Kreger, D. Lyerly, and R. H. Wagner. 1981. The effect of extracellular proteases from Gram-negative bacteria on the interaction of von Willebrand factor with human platelets. J. Lab. Clin. Med. 97:379-389.

25. Fox, J. E. B. 1985. Identification of actin-binding protein as the protein linking the membrane skeleton to glycoproteins on platelet plasma membranes. J. Biol. Chem. 260:11970-11977.

26. Berndt, M. C., C. Gregory, B. H. Chong; H. Zola, and P. A. Castaldi. 1983. Additional glycoprotein defects in Bernard-Soulier's syndrome: confirmation of genetic basis by parental analysis. Blood. 62:800-807.

27. Nurden, A. T., D. Dupuis, T. J. Kunicki, and J. P. Caen. 1981. Analysis of the glycoprotein and protein composition of Bernard-Soulier platelets by single and two-dimensional sodium dodecyl sulfatepolyacrylamide gel electrophoresis. J. Clin. Invest. 67;1431-1440.

28. Solum, N. O., I. Hagen, C. Filion-Myklebust, and T. Stabaek. 1980. Platelet glycocalicin: its membrane association and solubilization in aqueous media. Biochim. Biophys. Acta. 597:235-246.

29. Solum, N. O., and T. M. Olsen. 1984. Glycoprotein Ib in the Triton-insoluble (cytoskeletal) fraction of blood platelets. Biochim. Biophys. Acta. 799:209-220.

30. Taylor, M. A., and D. J. Anstee. 1984. The use of functional and quantitative assays to study glycoprotein $\mathrm{Ib}$ in platelets stored under various in vitro conditions. Thromb. Haemostasis. 52:271-275.

31. George, J. N. 1976. Platelet membrane glycoproteins: alterations during storage of human platelet concentrates. Thromb. Res. 8:719-724.

32. Bolin, R. B., F. Medina, and B. A. Cheney. 1981. Glycoprotein changes in fresh vs. room temperature-stored platelets and their buoyant density cohorts. J. Lab. Clin. Med. 98:500-510. 